\title{
ON ALGEBRAS OF OPERATORS WITH TOTALLY ORDERED LATTICE OF INVARIANT SUBSPACES
}

\author{
JOHN B. CONWAY 1
}

\begin{abstract}
For a Hilbert space $\mathfrak{H}$, let $Q$ be a weakly closed algebra of bounded operators on $\mathcal{H C}$ which contains the identity. $Q$ is said to be transitive if no closed subspace of $\mathcal{H C}$ is invariant under $Q$. There are no known proper subalgebras of $B(\mathcal{H C})$ which are transitive. In this paper it is shown that the only transitive algebra which satisfies a certain condition $\beta$ is $B(\mathcal{H C})$. Furthermore, a generalization of condition $\beta$ is given which characterizes those algebras with totally ordered lattice of invariant subspaces that are reflexive.
\end{abstract}

Let $\mathfrak{H C}$ be a Hilbert space and let $B(\mathcal{H C})$ be the algebra of bounded operators on $\mathfrak{F}$. Let $Q$ be a subalgebra of $B(\mathcal{H C})$ which contains the identity and which is closed in the weak operator topology (WOT). We denote by Lat $Q$ the lattice of subspaces of $\mathcal{H}$ which are invariant under all the operators in $Q$. Also, if $\mathscr{L}$ is a lattice of subspaces of $\mathfrak{H C}$, we denote by Alg $\&$ the algebra of all operators which leave these subspaces invariant. An algebra $Q$ is called reflexive if $a=A$ lg Lat $Q$. By means of von Neumann's double commutant theorem and some basic results in the theory of von Neumann algebras, every WOT closed selfadjoint algebra is reflexive. Hence, the search for reflexive algebras is a search for algebras which satisfy a version of the double commutant theorem.

In [4] Radjavi and Rosenthal studied algebras $a$ for which Lat $a$ is totally ordered and proved in this case that $a$ is reflexive if and only if $Q$ contains a masa (maximal abelian subalgebra of $B(\mathfrak{H C})$ ). In this paper we will examine other necessary and sufficient conditions for such algebras to be reflexive.

If $\Re \in$ Lat $Q$ and $P$ is the orthogonal projection onto $\Re$ then $Q P$ is the subalgebra of $B(\mathscr{T})$ consisting of all restrictions of elements of $a$ to $\mathfrak{T}$. We say that $a$ satisfies condition $\alpha$ if for each $\mathfrak{T}$ in Lat $a$ the only linear operators defined on a dense $a$ invariant manifold of $\mathfrak{T}$ (and taking values in $\mathfrak{T}$ ) which commute with members of $a P$ are the multiples of the identity.

Received by the editors April 8, 1970.

AMS 1969 subject classifications. Primary 4665, 4735; Secondary 0630.

Key words and phrases. Algebra of operators, reflexive algebra, transitive algebra, lattice of invariant subspaces.

1 The author gratefully acknowledges the support of the National Science Foundation.

Copyright @ 1971, American Mathematical Society 
Now let $M$ be any subspace of $\mathfrak{H}$; let $Q$ be a fixed algebra. We define the annihilator of $M$ by

$$
\text { ann } M=\{A \in a: A x=0 \text { for all } x \in M\} .
$$

It is easy to see that ann $M$ is a WOT closed left ideal of $a$. If $g$ is a left ideal of $a$ then we define the kernel of $\mathcal{g}$ by

$$
\text { ker } \mathfrak{d}=\{x \in \mathfrak{F}: A x=0 \text { for each } A \text { in } \mathscr{g}\} \text {. }
$$

Clearly ker $\mathcal{J}$ is a subspace of $\mathfrak{H C}$ and $M \subset$ ker ann $M$. We say that $a$ satisfies condition $\beta$ if for each finite dimensional subspace $M$ of Fe, $M=\operatorname{ker}$ ann $M$.

REMARK. Condition $\beta$ was inspired by a presentation of the ArtinWedderburn Theorem due to J. Tate which can be found in the article by Artin [1].

A special case of an algebra with a totally ordered lattice is a transitive algebra; that is, an algebra with Lat $Q=\{(0), \mathfrak{H}\}$. Arveson [2] proved that a transitive algebra which contains a masa is $B(\mathcal{H C})$, and hence is reflexive. In fact, his paper was the inspiration for the Radjavi-Rosenthal Theorem as well as the curiosity of the present author.

We can now state and prove the following theorem for transitive algebras which will be the archetype for our results on algebras with totally ordered lattice.

THEOREM A. Let a be a WOT closed transitive subalgebra of $B(\mathcal{H C})$ which contains the identity. Then the following are equivalent:

(a) $a$ is reflexive (and hence is $B(\mathcal{B C})$ );

(b) a contains a masa;

(c) a satisfies condition $\alpha$;

(d) a satisfies condition $\beta$.

Proof. That (a) implies (b) is clear. To prove that (b) implies (c) let $T$ be an operator densely defined on $\mathcal{H C}$ which commutes with $a$. Then $T$ has a (possibly unbounded) normal closure [2, p. 643], which we will also denote by $T$. It follows from Fuglede's Theorem [3] that the spectral projections of $T$ also commute with $a$. But since Lat $a$ $=\{0, \mathfrak{F}\}$ these projections can only be 0 or $I$. Thus, $T=\lambda I$ for some scalar $\lambda$.

(c) implies (d). We proceed by induction; let $x \neq 0$ and $y \in \operatorname{ker}$ ann $[x]$ (for $S \subset \mathfrak{K},[S]$ is the closed linear subspace spanned by $S$ ). Then $x \in[a x] \in$ Lat $a$ so that $[a x]=\mathcal{F}$. Also, $y \in$ ker ann $[x]$ means that if $A \in Q$ and $A x=0$ then $A y=0$. Thus if we set $T(A x)=A y$ for each $A$ in $Q, T$ is a densely defined linear operator which is easily seen to 
commute with $Q$. Thus, there is a scalar $\lambda$ with $A y=\lambda A x$ for each $A \in a$. In particular, by taking $A=I$ we get $y=\lambda x \in[x]$, so ker ann $[x]$ $=[x]$ for $x \neq 0$.

Now suppose we have ker ann $N=N$ for all $n$ dimensional spaces $N$ and let $M$ be $(n+1)$-dimensional. Let $N$ be any $n$ dimensional subspace of $M$ and let $x \in M$ with $x \notin N$. Put $\mathcal{g}=$ ann $N$; then ann $M$ $=\mathscr{J}$ hann $[x]$ and $\operatorname{ker}$ ann $M=\operatorname{ker}(g \cap$ ann $[x])$.

Since $d$ is a left ideal of $Q$ we have $[\delta x] \in$ Lat $Q$. If $[\delta x]=(0)$ then $x \in$ ker $\mathcal{J}=N$ which is false. Thus, $[\mathcal{J} x]=\mathfrak{F C}$. Fix $y \in \operatorname{ker}$ ann $M$. If $A \in \mathcal{J}$ and $A x=0$ then $A \in$ ann $M$ and so $A y=0$. Thus $T(A x)=A y$ for $A \in \mathcal{J}$ is densely defined and commutes with $Q$. By (c) we have a scalar $\lambda$ so that $\lambda A x=A y$ for each $A$ in $\mathcal{J}$. This says that $y-\lambda x \in \operatorname{ker} \mathcal{d}$ $=N$ or $y \in M$.

(d) implies (a). We show that $Q$ is WOT dense in $B(\mathfrak{C})$. That is, let $\left\{x_{1}, \cdots, x_{n}\right\}$ be linearly independent in $\mathcal{H C}$ and $\left\{y_{1}, \cdots, y_{n}\right\}$ $\subset \mathcal{K}$; we will show that for each $\epsilon>0$ there is an $A \in Q$ with $\left\|A x_{j}-y_{j}\right\|$ $<\epsilon$ for $1 \leqq j \leqq n$. Let $M_{j}$ be the span of $\left\{x_{i}: i \neq j\right\}$. Then $x_{j} \notin M_{j}$ $=\operatorname{ker}$ ann $M_{j}$; thus there is a $B_{j} \in$ ann $M_{j}$ with $B_{j} x_{j} \neq 0$. Since $a$ is transitive there is an $A_{j} \in Q$ with $\left\|A_{j} B_{j} x_{j}-y_{j}\right\|<\epsilon$. Let $A$ $=A_{1} B_{1}+\cdots+A_{n} B_{n} \in Q$. It is easy to check that $A$ is the desired operator. This completes the proof.

Conditions $\alpha$ and $\beta$ are not equivalent to reflexivity if we do not assume that $a$ is transitive. For example if $a$ is the von Neumann algebra of all multiplication operators on $\mathcal{F}=L^{2}(0,1)$ and $x$ is the identically one function then $\operatorname{ker}$ ann $[x]=\mathscr{T}$. Even if we assume that Lat $Q$ is totally ordered it does not follow that conditions $\alpha$ and $\beta$ imply reflexivity. For example let $\alpha$ be the algebra consisting of all $3 \times 3$ matrices

$$
\left(\begin{array}{lll}
a & 0 & 0 \\
c & a & 0 \\
d & e & b
\end{array}\right)
$$

where $a, b, c, d, e$ are arbitrary scalars. Then $a$ has totally ordered lattice, satisfies conditions $\alpha$ and $\beta$, but is not reflexive.

Before formulating the result for algebras with totally ordered lattice let us first state an easy lemma without proof.

Lemma 1. Suppose Lat $a$ is totally ordered; let $\mathfrak{T} \in$ Lat $a$ and let $P$ be the orthogonal projection on $\mathfrak{M}$. Then:

$$
\text { Lat }(a P)=\{\Re: \Re \in \text { Lat } a \text { and } \Re \subset \mathfrak{N}\} .
$$

If $B$ is a masa contained in $a$ then $P \in B$ and $B P$ is a masa contained 
in $a P$. (b) If we consider $P^{\perp} Q P^{\perp} \subset B\left(N^{\perp}\right)$ then $\operatorname{Lat}\left(P^{\perp} a P^{\perp}\right)$ $=\{\Re \ominus \Re: \Re \in$ Lat $a$ and $\Re \supset \Re\}$. If $B$ is a masa contained in $a$ then $\mathrm{B}^{\perp}$ is a masa in $B\left(\Re^{\perp}\right)$.

If Lat $a$ is totally ordered but finite then it would seem that Theorem A could be formulated and proved by considering the algebra induced by $a$ on $\mathfrak{F C} / \mathbb{T}$ for each $\mathscr{T C}$ in Lat $a$. This is indeed the case even if Lat $a$ is not finite. However we will work with the algebras $P^{\perp} a P^{\perp}$ instead of these induced algebras. Say that $a$ satisfies condition $\alpha$ (or respectively, condition $\beta$ ) on quotients if and only if for each $\mathfrak{M} \in$ Lat $a, P^{\perp} Q P^{\perp} \subset \otimes\left(M^{\perp}\right)$ satisfies condition $\alpha$ (resp., condition $\beta$ ). We can now state the main result.

TheOrem B. Let a be a WOT closed subalgebra of $B(\mathfrak{H C})$ containing the identity and with Lat a totally ordered. Then the following are equivalent:

(a) a is reflexive;

(b) a contains a masa;

(c) a satisfies condition $\alpha$ on quotients and a contains the orthogonal projections on the members of Lat $Q$;

(d) a satisfies condition $\beta$ on quotients.

Before proving this theorem we will give some preliminary lemmas. If $n$ is an integer then let $\mathfrak{H}^{(n)}$ be the direct sum of $n$ copies of $\mathfrak{F}$. Let $A^{(n)}=\sum_{j=1}^{n} \oplus A_{j}$ where $A_{j}=A$. Define $Q^{(n)}=\left\{A^{(n)}: A \in Q\right\} \subset B\left(\mathcal{F C}^{(n)}\right)$. A proof of the following well-known lemma can be found in [5, p. 684].

Lemma 2. If $a$ is a weakly closed subalgebra of $B(\mathfrak{H C})$ containing the identity and $B \in B(\mathcal{H C})$ is such that Lat $\mathbb{Q}^{(n)} \subset$ Lat $B^{(n)}$ for each $n \geqq 1$ then $B \in Q$.

Lemma 3. Let a satisfy condition $\beta$ and suppose $B \in B(\mathcal{H C})$ is such that Lat $Q^{(k)} \subset$ Lat $B^{(k)}$ for $1 \leqq k \leqq n$. If $\Re \in$ Lat $Q^{(n+1)}$ does not meet at least one of the coordinate spaces then $M \in L$ Lat $B^{(n+1)}$.

Proof. We may assume that $\mathfrak{T}$ misses the last coordinate space, i.e., $\mathfrak{T} \cap\left(\mathfrak{H}^{(n)} \oplus(0)\right)=(0)$. Let $\left(x_{1}, \cdots, x_{n+1}\right) \in \mathscr{N}$; we must show that $\left(B x_{1}, \cdots, B x_{n+1}\right) \in \mathscr{T}$. We can assume without loss of generality that $\mathbb{N}=\left[Q^{(n+1)}\left(x_{1}, \cdots, x_{n+1}\right)\right]$. Since $\mathfrak{M}$ misses the last coordinate, if $A \in Q$ satisfies $A x_{1}=\cdots=A x_{n}=0$ then $A x_{n+1}=0$. That is, ann $\left[x_{1}, \cdots, x_{n}\right] \subset$ ann $\left[x_{n+1}\right]$, so that by condition $\beta$ we get $x_{n+1}$ $\in\left[x_{1}, \cdots, x_{n}\right]$. Hence there are scalars $c_{1}, \cdots, c_{n}$ such that $x_{n+1}$ $=c_{1} x_{1}+\cdots+c_{n} x_{n}$. Therefore, 


$$
\mathfrak{M}=\left[\left(A x_{1}, \cdots, A x_{n}, \sum_{j} c_{j} A x_{j}\right): A \in Q\right] .
$$

If we put

$$
\mathfrak{T}_{1}=\left\{\left(y_{1}, \cdots, y_{n}\right):\left(y_{1}, \cdots, y_{n+1}\right) \in \mathscr{T} \text { for some } y_{n+1} \in \mathcal{K}\right\},
$$

then $\mathfrak{T}_{1}$ is closed in $\mathfrak{F}^{(n)}$ and $\mathfrak{T}_{1} \in$ Lat $Q^{(n)}$. By hypothesis we get $\mathfrak{M}_{1} \in$ Lat $B^{(n)}$. Hence, $\left(B x_{1}, \cdots, B x_{n}\right) \in \mathscr{N}_{1}$ and so $\left(B x_{1}, \cdots, B x_{n+1}\right)$ $=\left(B x_{1}, \cdots, B x_{n}, \sum_{j} c_{j} B x_{j}\right) \in \mathfrak{M}$, completing the proof of the lemma.

REMARK. Those familiar with Radjavi and Rosenthal [4] will have noticed a similarity between the preceding lemma and their Lemma 3. Also the strategy we will employ in proving that (d) of Theorem B implies (a) is theirs [4, p. 688].

Proof of Theorem B. The proof that (a) implies (b) can be found in $[4$, p. 691].

(b) implies (c). If $\Re \in$ Lat $Q$ and $P$ is the orthogonal projection of

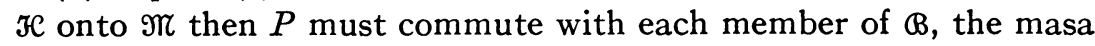
in $a$. Hence each $P$ is in $B C Q$.

By Lemma $1, P^{\perp} a P^{\perp}$ contains a masa and has totally ordered lattice. If we can show that such algebras satisfy condition $\alpha$, we will be done. Again $Q P$ (by Lemma 1) also satisfies these conditions; so if we can prove that an operator $T$ defined on a dense $Q$ invariant manifold of $\mathfrak{H}$ which commutes with $Q$ is a multiple of the identity then we will be done. But such a $T$ has normal closure [2, p. 643] and, as in the proof of Theorem A, the spectral projections must commute with $a$. Since Lat $a$ is totally ordered $T$ must be a multiple of the identity.

(c) implies (d). We will show that condition $\alpha$ implies condition $\beta$; with this and Lemma 1 we will have proven this implication. We proceed by induction on the dimension of $M \subset \Re$.

Let $x \neq 0$ be any vector in $\mathcal{H}$ and let $y \in$ ker ann $[x]$. That is, $A \in Q$ and $A x=0$ implies that $A y=0$. If $\mathscr{T}=[a x]$ and $P$ is the orthogonal projection on $\Re \in \in$ Lat $a$ then $P \in Q$ by hypothesis. Hence $P^{\perp} x=0$ implies $P^{\perp} y=0$; or, $y \in \mathfrak{M}$. (Remark: The only place where the additional hypothesis in (c) is needed is to get $y$ in $\mathfrak{T}$ and a similar fact later in this proof. It seems likely that the only part of (c) needed is that $Q$ satisfies condition $\alpha$.) If we set $T(A x)=A y$ for $A$ in $Q$ then $T$ is a well-defined linear operator from $a x$ into $a y \subset \mathfrak{T}$. Also $T$ commutes with $Q$. Hence, there is a scalar $\lambda$ with $T=\lambda I$; that is, $A y=\lambda A x$ for each $A \in Q$. In particular, we get $y=\lambda x \in[x]$, so $[x]=\operatorname{ker}$ ann $[x]$.

Now suppose ker ann $M=M$ whenever $M$ has dimension $n$. Let $M$ be $n$ dimensional and $x \notin M$. Put $\mathcal{J}=$ ann $M$; then $\mathfrak{T}=[\delta x] \neq(0)$ by 
the induction hypothesis and $\mathfrak{T} \in$ Lat $a$ since $\mathcal{J}$ is a left ideal of $a$. Let $P$ be the orthogonal projection on $\mathfrak{T}$. If $A \in \mathcal{J}=$ ann $M$ then $P^{\perp} A \in \operatorname{ann}[M, x]$. So if $y \in \operatorname{ker}$ ann $[M, x]$ then $A \in \mathcal{J}$ implies $P^{\perp} A y$ $=0$; that is $A y \in \mathfrak{I}=[\mathcal{J} x]$. Therefore if we let $T(A x)=A y$ for $A \in \mathcal{J}$ then, as before, it follows that $T=\lambda I$ for some scalar $\lambda$. Thus, $A(y-\lambda x)=0$ for each $A \in \mathcal{J}$; or, $y-\lambda x \in$ ker ann $M=M$ so that $\operatorname{ker}$ ann $[M, x]=[M, x]$.

(d) implies (a). Let $B \in B(\mathfrak{F})$ and suppose Lat $a \subset$ Lat $B$. By Lemma 2 we must show that Lat $\mathbb{Q}^{(n)} \subset$ Lat $B^{(n)}$ for each integer $n \geqq 1$; we proceed by induction. The case $n=1$ is the hypothesis on $B$ so we may assume Lat $Q^{(k)} \subset$ Lat $B^{(k)}$ for $k=1, \cdots, n$. Let $\Re \in$ Lat $Q^{(n+1)}$; if $\Re$ does not meet one of the coordinate spaces then, by Lemma 3 , we have $\mathfrak{M} \in$ Lat $B^{(n+1)}$. Now suppose $\mathfrak{N}$ meets each coordinate space of $\mathfrak{F}^{(n+1)}$. As in $\left[4\right.$, p. 689], for $1 \leqq i \leqq n+1$, let $\mathfrak{T}_{i}$ be the subspace of $\mathfrak{H C}$ consisting of all vectors $x$ such that $\mathscr{T}$ contains an element all of whose entries are zero except the $i$ th which is $x$. Then each $\mathfrak{M}_{i} \in$ Lat $Q$ and $\mathscr{N}=\bigcap_{i=1}^{n+1} \mathfrak{M}_{i}$ is the smallest of these subspaces. Let $P$ be the projection on $\mathfrak{x}, Q=P^{(n+1)}, \hat{a}=P^{\perp} Q P^{\perp}$, $\hat{\mathfrak{C}}$ $=\mathfrak{H} \ominus \Re=P^{\perp} \mathfrak{H C}, \hat{B}=P^{\perp} B P^{\perp}$. Then Lat $\hat{Q}^{(k)} \subset$ Lat $\hat{B}^{(k)}$ for $1 \leqq k \leqq n$. Also $Q^{\perp} \mathscr{T} \in$ Lat $\hat{Q}^{(n+1)}$ and $Q^{\perp} \mathscr{T}$ fails to meet one of the coordinate spaces of $\hat{\mathfrak{C}}^{(n+1)}$. Since, by (d), $\hat{\mathbb{Q}}$ satisfies condition $\beta$, we have that $Q^{\perp} \mathbb{N} \in$ Lat $\hat{B}^{(n+1)}$ by Lemma 3. It follows that $\Re \in$ Lat $B^{(n+1)}$. This completes the proof of Theorem B.

\section{BIBLIOGRAPHY}

1. E. Artin, The influence of J.H. M. Wedderburn on the development of modern algebra, Bull. Amer. Math. Soc. 56 (1950), 65-72.

2. W. B. Arveson, $A$ density theorem for operator algebras, Duke Math. J. 34 (1967), 635-647. MR 36 \#4345.

3. B. Fuglede, A commutativity theorem for normal operators, Proc. Nat. Acad. Sci. U.S.A. 36 (1950), 35-40. MR 11, 371.

4. H. Radjavi and P. Rosenthal, On invariant subspaces and reflexive algebras, Amer. J. Math. 91 (1969), 683-692. MR 40 \#4796.

5. D. Sarason, Invariant subspaces and unstarred operator algebras, Pacific J. Math. 17 (1966), 511-517. MR 33 \#590.

INDIANA University, BLOOMINGTON, INDIANA 47401 